\title{
Acquired factor VIII inhibitor associated with chronic untreated hepatitis C
}

\author{
Abdussalam Shredi MD, Benjamin W. Elberson PhD, Saif EI Nawaa MD, Amr Ismail MD
}

\begin{abstract}
Acquired inhibitors of coagulation are antibodies that either inhibit the activity or increase the clearance of a clotting factor. A hemorrhagic diathesis is a common clinical manifestation in affected patients. Acquired factor VIII inhibitor - or acquired hemophilia $A$ - is a rare disorder and presents similarly to hemophilia $A$, though patients are less likely to develop hemarthroses. This inhibition is most commonly due to autoantibodies against coagulation factor VIII. These autoantibodies often occur in pregnancy, autoimmune disorders, solid tumors, and lymphoproliferative syndromes. Several drugs, including penicillins, phenytoin, and sulfa drugs, have also been associated with antibodies to factor VIII. Chronic infection with the hepatitis $C$ virus (HCV), in addition to various degrees of liver inflammation and fibrosis, can have extrahepatic manifestations, especially autoimmune disorders. The most common hematological complications of HCV infection are thrombocytopenia, cryoglobulinemia, and anti-cardiolipin antibodies. A few cases of factor VIII inhibitors occurring in HCV patients have been reported, with a higher incidence after prolonged treatment with interferon- $\alpha$. Here, we present a case of a patient with chronic untreated HCV infection developing acquired factor VIII deficiency.
\end{abstract}

Keywords: Factor VIII inhibitor, hepatitis C, hematoma, acquired hemophilia A, autoimmune hemophilia

\section{INTRODUCTION}

Acquired factor VII inhibitor - or acquired hemophilia A (AHA) - has an annual incidence estimated between 0.2 and 4 per million persons per year and is considered a rare coagulation disorder. ${ }^{1,2}$ The development of AHA is often idiopathic without an obvious cause. ${ }^{3}$ However, when a cause is identified, AHA is often associated with interferon- $\alpha$ exposure, or with concurrent X-linked hemophilia A, cancer, autoimmune disease, HIV, pregnancy, or other immune-modulating conditions and treatments. ${ }^{3-8}$ As

Corresponding author: Abdussalam Shredi Contact Information: Abdussalam.Shredi@ttuhsc.edu DOI: $10.12746 /$ swrccc.v6i23.465 discussed by Franchini, et al. in their review of AHA, the median age for diagnosis is between 60 and 80 years old - male predominant - with another significant population in female patients between ages 20 and 30 due to the $10 \%$ of AHA cases occurring in the post-partum period. ${ }^{2}$ The development of acquired hemophilia A without previous drug exposure and without genetic hemophilia is rare, though it has been described previously by Sugishita, et al. in a report of an older man with chronic hepatitis $C,{ }^{9}$ a case similar to the one presented here. Despite the rarity of AHA precluding large-scale studies, remission is achievable using chemotherapeutic-based treatment plans. ${ }^{10}$ However, the all-cause mortality rate for patients who have developed AHA remains high at $21 \%$, based on a meta-analysis of 359 cases. ${ }^{10}$ The development of clinically significant symptoms is positively correlated with factor VIII inhibitor levels, with some 
low-titer cases suspected to go unnoticed without a significant inciting bleeding event. ${ }^{2}$ These cases are found in $15 \%$ of the known patient population with acquired factor VIII inhibitors and depend upon the subtype of autoantibody and the domain of factor VIII that they recognize. ${ }^{2}$ We present a case of a 61-yearold woman with chronic untreated hepatitis $C$ who developed acquired hemophilia $A$, which ultimately proved fatal.

\section{CASE}

A 61-year-old woman with a past medical history of liver cirrhosis secondary to chronic untreated hepatitis $C$ presented with severe lower extremity pain and swelling beginning one day prior and worsening. The patient also reported a history of easy bruising for 2 months before this presentation.

Her initial vital signs were stable. Labs revealed hemoglobin $7.0 \mathrm{gm} / \mathrm{dL}$, hematocrit $20.9 \%$, platelets $217 \mathrm{k} / \mu \mathrm{L}$, prothrombin time (PT) 10.1, international normalized ratio (INR) 0.91 , partial thromboplastin time (PTT) 67.2, not corrected by a mixing study, and D-dimer $2932 \mathrm{ng} / \mathrm{mL}$. Her lactate dehydrogenase was elevated at 542 units/L, her haptoglobin was low at $<15 \mathrm{mg} / \mathrm{dL}$, and there was no lab evidence for iron, folate, or $\mathrm{B}_{12}$ deficiency. She had a high viral load (>4 million IU/mL) of hepatitis C virus, genotype 2 .

Further studies to determine a possible factor deficiency suggested by her clinical presentation and medical history were done. Thrombosis was ruled out, but multiple sites of bleeding were identified. The factor VIII level was low at $<5 \%$, factor IX was $113 \%$, factor XI was $86 \%$, and factor XII was $61 \%$. Factor VIII inhibitor was detected at titer of over 854.4 Bethesda units/L (BU/L), performed at Quest Diagnostics Nichols Institute, San Juan Capistrano, CA. No other immunoglobulin abnormalities were detected. The antinuclear antibody was positive, the rheumatoid factor was high at $54 \mathrm{IU} / \mathrm{L}$. Complement 3 and 4 were low at $4 \mathrm{mg} / \mathrm{dL}$ and $2 \mathrm{mg} / \mathrm{dL}$, respectively. Factor VIII levels, PTT, platelet counts, and anti-factor VIII antibodies were tracked to monitor her treatment responses (Figure 1). After these studies were drawn but before they could be reported, the patient left the hospital against medical advice. She was re-admitted 17 days later. Based on these laboratory studies, the patient was diagnosed with acquired factor VIII inhibitor coagulopathy. Coagulation studies were used to monitor her progression, primarily relying on PTT. She was started on $\mathrm{rFVII}$, prednisolone $1 \mathrm{mg} / \mathrm{kg} /$ day $(60$ $\mathrm{mg}$ ), and cyclophosphamide $2 \mathrm{mg} / \mathrm{kg} /$ day $(120 \mathrm{mg}$ ).

The prednisolone was increased to $80 \mathrm{mg}$ daily, and intravenous recombinant human coagulation factor VIla (rFVIla, NovoSeven) $4.8 \mathrm{mg}$ daily was added as recommended by $\mathrm{Ma}$ and Carrizosa in patients with poor initial response and a Bethesda assay titer $>5 \mathrm{BU} / \mathrm{L} .{ }^{11}$ The patient also received a single intravenous dose of anti-CD21 monoclonal antibodies (rituximab, Rituxan) at $375 \mathrm{mg} / \mathrm{m}^{2}$. A medication timeline is illustrated in Figure 2. After a week of treatment, the muscle swelling decreased, and the hematuria stopped. Twelve days after admission, the partial thromboplastin time was $53.0 \mathrm{sec}$, and the factor VIII level was $9 \%$, indicating partial recovery.

Despite best efforts to address bleeding events and hepatitis $C$ treatment with sofosbuvir $400 \mathrm{mg}$ and velpatasvir $100 \mathrm{mg}$ (Epclusa), she continued to have intermittent bleeding and then started to acutely deteriorate. She became severely leukopenic, thrombocytopenic, and anemic $(\mathrm{Hb}<7 \mathrm{gm} / \mathrm{dL})$. Her chest $\mathrm{x}$-ray then suggested early acute respiratory distress syndrome. The patient died this same day.

\section{Discussion}

Chronic HCV infection primarily affects the liver but can have extrahepatic manifestations, including arthralgias, skin manifestations, xerostomia, exophthalmia, and sensory neuropathy. The main biological abnormalities observed in chronic HCV infection are mixed cryoglobulinemia and the presence of autoantibodies. These antibodies include anti-nuclear antibody, rheumatoid factor, anti-cardiolipin, antithyroglobulin, and anti-smooth muscle cell antibody. Therefore, it is not unexpected that autoantibodies to factor VIII occur in patients infected with the hepatitis $\mathrm{C}$ virus. As the development of AHA is most likely linked to the presence of a chronic active hepatitis $C$ infection, treating the $\mathrm{HCV}$ in addition to managing 


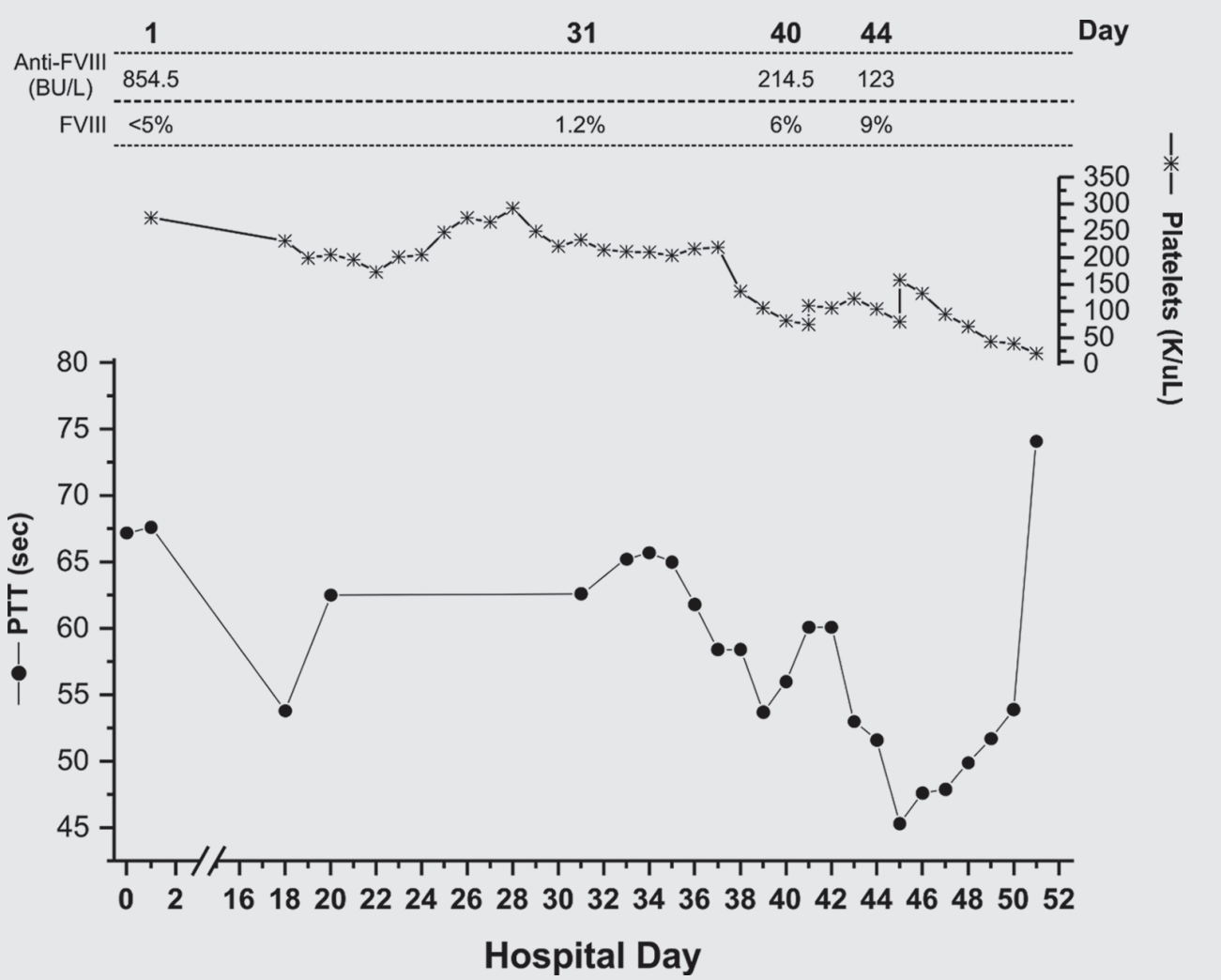

Figure 1. PTT, Platelet, Factor VIII, and anti-Factor VIII antibody levels during the hospitalization.

The patient's clinical condition can be tracked using platelet and PTT values. Despite the improvement in FVIII and anti-FVIII levels, she decompensated due to frequent bleeding events and could not be stabilized. The gap from day 2 to day 16 was due to the patient leaving the hospital against medical advice after undergoing initial testing. These data support the efficacy of described treatments for AHA and emphasize the high mortality risk despite best treatment efforts. Earlier identification of AHA in this patient may have allowed a better response from more prompt treatment.

symptoms is a reasonable approach to controlling the development of factor VII autoantibodies. ${ }^{2}$ This is especially true when the primary method of directed AHA treatment, based on chemotherapeutic medications causing immunosuppression, is likely to cause increased viral replication and disease exacerbation in the setting of chronic hepatitis C infection. ${ }^{12,13}$ Unfortunately, this patient's access to care was limited by late insurance approval, preventing her from receiving sofosbuvir and velpatasvir (Epclusa) until late in her disease course (Figure 2).
Bleeding episodes in AHA are unpredictable; they may affect a wide range of tissues and occur without obvious insult or known hereditary coagulopathy. ${ }^{14}$ The propensity to develop factor VIII antibodies is not inherited and occurs in men and women with equal frequency with a peak incidence between 60 and 67 years, becoming increasingly common with older age though a wide age range has been reported..$^{1,11}$ This prevalence of extra-articular bleeding is a point of distinction between congenital and acquired hemophilia; hemarthrosis is not typically the presenting 


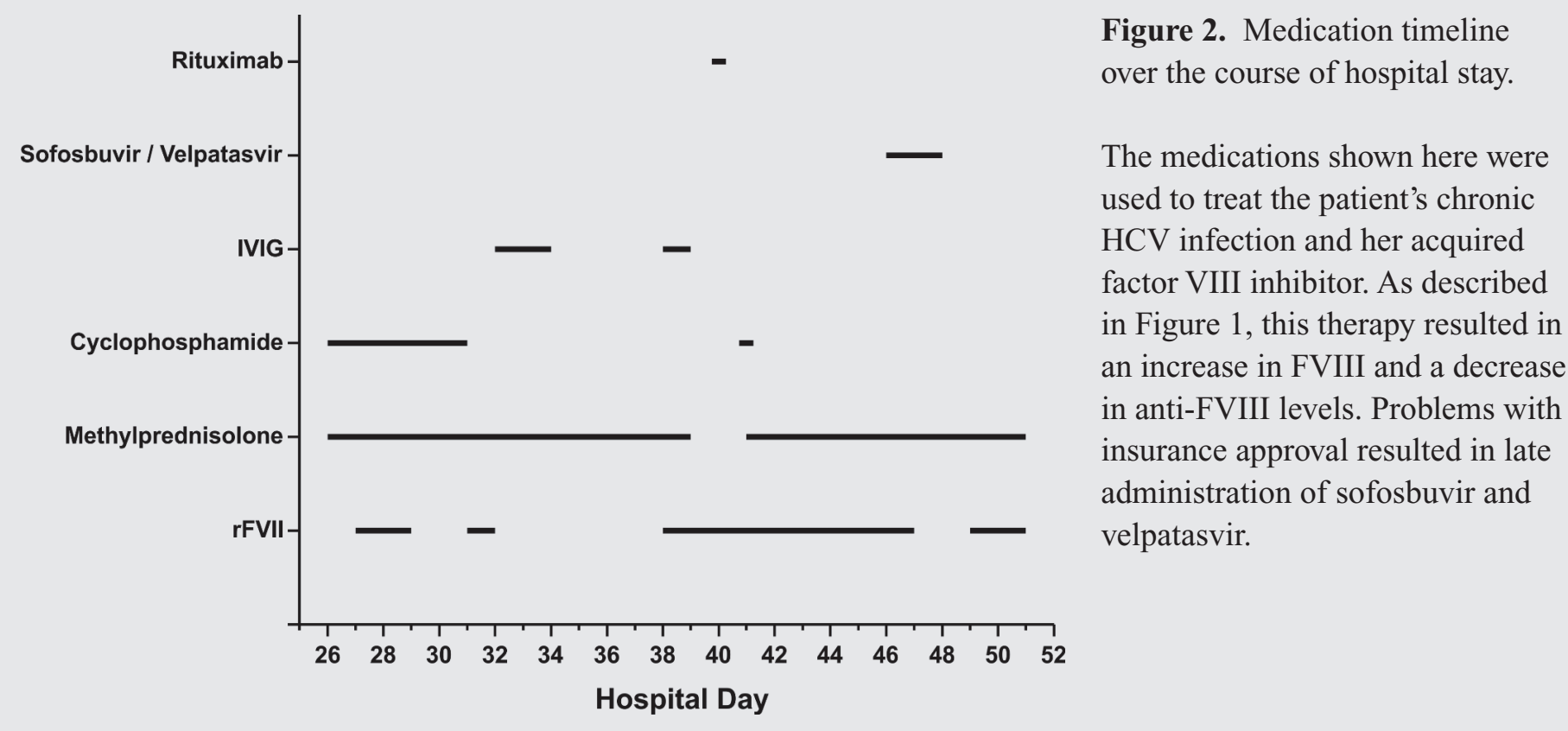

symptom in acquired hemophilia but is in congenital hemophilia. ${ }^{1,11}$

Laboratory evaluation of AHA includes measurement of a prolonged activated PTT and a normal PT without thrombocytopenia. ${ }^{11,15}$ However, multiple other conditions may present similarly: factor VIII, IX, or XI deficiencies without autoantibody production or von Willebrand disease. To differentiate between these diseases, a mixing study should be performed. ${ }^{11,15}$ If no correction is observed, an inhibitor is likely pathologically involved. While antiphospholipid syndrome would cause a lack of correction as well as similar laboratory results, the patient would experience thrombosis rather than bleeding. The Bethesda assay is currently the gold standard for diagnosis and tracking of treatment responses in AHA..$^{11,15}$

Treating factor VIII autoantibodies should address acute bleeding by raising factor VIII levels or bypassing this point in the coagulation cascade - as was attempted in this patient - as well as long-term risk mitigation using immunosuppression to eliminate the autoantibody. ${ }^{11,15} \mathrm{Ma}$ and Carrizosa suggest the use of desmopressin to raise factor VIII, a direct infusion of factor VIII, or an infusion activated factor VII as a bypass. ${ }^{11}$ Even with recommended care, mortality in patients with acquired hemophilia ranges between 8 and 22\%, depending on underlying conditions, with a high rate of severe bleeding events. ${ }^{1}$ Autoantibody titers in acquired factor VIII deficiency positively correlate with mortality; thus elimination of the inhibitor is a reasonable approach to prevent clinical decline. ${ }^{11}$ Depending on the patient, their comorbidities, and their unique response to treatment, some combination of glucocorticoids, cyclophosphamide, and rituximab has the best results in AHA, with glucocorticoids and cyclophosphamide having a significant effect in $60-70 \%$ of patients. ${ }^{11}$ Cyclophosphamide appears to be one of the most successful drugs for treatment of this condition. If immunosuppression is unsuccessful or not tolerated, IVIG may be used to directly antagonize the autoantibody, with a reported efficacy in $30 \%$ of patients. ${ }^{11}$ As detailed above, we attempted multiple approaches to manage this patient's acquired factor VIII deficiency but were ultimately unsuccessful.

Reports of AHA in the setting of chronic untreated $\mathrm{HCV}$ infection, especially without prior administration 
of interferon, are exceedingly rare. Our understanding of acquired factor VII inhibitors is inadequate due to low case volume, especially when no predisposing immunological conditions are present or immune-modulating treatments were used. Time to diagnosis and treatment initiation is essential for a good outcome, but this can be difficult due to the lack of a range of effective medications and the rarity of this condition. Although we used several therapeutic approaches, plasmapheresis was not attempted with this patient, and her HCV infection was not addressed until late in her disease course. These are two treatments might have provided a better outcome. More studies to quickly identify and treat AHA are necessary if we are to reduce the high mortality rate of this condition.

\section{Key STATEMENTS}

1. This case illustrates the difficulty in the management of bleeding associated with acquired Factor VIII antibodies.

2. Prospective studies in patients with hepatitis $C$ infections could clarify the incidence and clinical significance of these antibodies.

Article citation: Shredi A, Elberson BW, El Nawaa S, Ismail A. Acquired factor VIII inhibitor associated with chronic untreated hepatitis $C$. The Southwest Respiratory and Critical Care Chronicles 2018;6(23):17-21

From: The Department of Internal Medicine at Texas Tech University Health Sciences Center in Lubbock, TX Submitted: $1 / 22 / 2018$

Accepted: $3 / 28 / 2018$

Reviewers: Catherine Jones MD, Kenneth Nugent MD Conflicts of interest: None

This work is licensed under a Creative Commons

Attribution-ShareAlike 4.0 International License.

\section{REFERENCES}

1. Franchini M, Lippi G. Acquired factor VIII inhibitors. Blood 2008;112:250-5.

2. Franchini M, Gandini G, Di Paolantonio T, Mariani G. Acquired hemophilia A: a concise review. Am J Hematol 2005;80:55-63.
3. Mulliez SM, Vantilborgh A, Devreese KM. Acquired hemophilia: a case report and review of the literature. Int J Lab Hematol 2014;36:398-407.

4. Franchini M, Capra F, Nicolini N, et al. Drug-induced anti-factor VIII antibodies: a systematic review. Med Sci Monit 2007;13:Ra55-61.

5. Castenskiold EC, Colvin BT, Kelsey SM. Acquired factor VIII inhibitor associated with chronic interferon-alpha therapy in a patient with haemophilia A. Br J Haematol 1994;87: 434-6.

6. Paul S, Javed U, Tevendale R, Lanford J, Liu R. Acquired factor VIII inhibitor in an HIV-infected patient after treatment with pegylated interferon-alpha 2a and ribavirin. Aids. England 2007:784-5.

7. Botianu AM, Demian S, Macarie I, Georgescu D, Oltean G, Bataga S. Acquired haemophilia complicated with gastrointestinal bleeding and spontaneous iliopsoas muscle haematoma in a woman with chronic $\mathrm{C}$ hepatitis under treatment with pegylated IFN alpha 2a and ribavirin. J Gastrointestin Liver Dis 2012;21:93-5.

8. Campos-de-Magalhaes M, Eduardo Brandao-Mello C, Lucia Elias Pires M, Cecilia da Fonseca Salgado M, Barcelo de Brito S, Jose de Almeida A. Factor VIII and IX deficiencies related to acquired inhibitors in a patient with chronic hepatitis $\mathrm{C}$ virus infection receiving treatment with pegylated interferon plus ribavirin. Hematology 2011;16:80-5.

9. Sugishita K, Nagase H, Takahashi T, Takenaka K, Shiratori Y, Omata M. Acquired factor VIII inhibitor in a non-hemophilic patient with chronic hepatitis $\mathrm{C}$ viral infection. Intern Med 1999;38:283-6.

10. Bitting RL, Bent S, Li Y, Kohlwes J. The prognosis and treatment of acquired hemophilia: a systematic review and meta-analysis. Blood Coagul Fibrinolysis 2009;20:517-23.

11. Ma AD, Carrizosa D. Acquired factor VIII inhibitors: pathophysiology and treatment. Hematology Am Soc Hematol Educ Program 2006:432-7.

12. Zignego AL, Giannini C, Gragnani L, Piluso A, Fognani E. Hepatitis $\mathrm{C}$ virus infection in the immunocompromised host: a complex scenario with variable clinical impact. J Transl Med 2012;10:158.

13. Sagnelli E, Sagnelli C, Pisaturo M, Coppola N. Hepatic flares in chronic hepatitis $\mathrm{C}$ : spontaneous exacerbation vs hepatotropic viruses superinfection. World J Gastroenterol 2014;20:6707-15.

14. Knoebl P, Marco P, Baudo F, et al. Demographic and clinical data in acquired hemophilia A: results from the European Acquired Haemophilia Registry (EACH2). J Thromb Haemost 2012;10:622-31.

15. Delgado J, Jimenez-Yuste V, Hernandez-Navarro F, Villar A. Acquired haemophilia: review and meta-analysis focused on therapy and prognostic factors. Br J Haematol 2003;121: 21-35. 